\title{
MODELO PARA EL ANÁLISIS DE EXPERIENCIAS DE INNOVACIÓN PÚBLICA: EL CASO DEL CENTRO DE INTEGRACIÓN CIUDADANA
}

\author{
Jesús Rubio Campos \\ El Colegio de la Frontera Norte, México \\ rubio@colef.mx \\ Marisol Calzada Torres \\ El Colegio de Sonora, México \\ nenufarlex@gmail.com
}

\section{RESUMEN}

El artículo analiza la creación y desarrollo del Centro de Integración Ciudadana (CIC) de San Pedro Garza García, Nuevo León, México. Este es un esfuerzo creado por la sociedad civil local, que en conjunto con otros actores, que colabora con distintos órdenes y dependencias del gobierno mexicano para la detección y solución de problemáticas sociales en el Área Metropolitana de Monterrey por medio de una participación ciudadana masiva. A partir de un trabajo de análisis documental, entrevistas en profundidad y cuestionarios a actores clave, el artículo destaca que el CIC enlaza exitosamente a ciudadanos y autoridades, al recibir, validar, canalizar y dar seguimiento a los reportes ciudadanos recibidos por medio de redes sociales digitales

Palabras clave: Innovación pública, Sociedad civil, Redes sociales digitales, tecnología social, Gobierno 2.0, México. 


\begin{abstract}
This article analyzes the creation and development of the Centro de Integración Ciudadana (CIC) of San Pedro Garza García, Nuevo León, Mexico. This is an endeavor created by the local civil society, which, together with other players, collaborates with different levels and agencies of the Mexican government for the detection and solution of social problems in the Metropolitan Area of Monterrey via massive citizen participation. Based on documentary analysis, in-depth interviews and questionnaires to key actors, the article highlights that the CIC successfully links together citizens and authorities, to receive, validate, channel and do follow-up on the citizen reports received via digital social networks.
\end{abstract}

Keywords: Public innovation, Civil society, Digital social networks, Social technology, Government 2.0, Mexico. 


\section{INTRODUCCIÓN}

La innovación pública surge dentro de los modelos de gestión pública de nueva gobernanza como una forma de atender los problemas sociales, involucrando no solo al gobierno, sino a la sociedad civil en general, así como a la iniciativa privada, en emprendimientos para la detección de necesidades, la definición conjunta de las problemáticas sociales y la co-creación de soluciones, haciendo uso intensivo de tecnologías de la información basadas en Internet.

El objetivo de la presente investigación es proponer un modelo de análisis de experiencias de innovación pública, a partir de una revisión de las teorías sobre el tema, de autores tales como Sørensen y Torfing (2012), Kallio, Lappalainen y Tammela (2013), Bäck et al. (2013), García (2014), Pardo (2014), Zubbrigen y González (2014), Santiago (2014), Mariñez (2015) y Rojas-Martín y Criado (2015).

Con el modelo propuesto, en la segunda parte se documenta y analiza un caso concreto de innovación pública, el del Centro de Integración Ciudadana (CIC), cuya base está en San Pedro Garza García Nuevo León, México, pero su zona de influencia operativa se expande a toda el Área Metropolitana de Monterrey y recientemente a las ciudades de Saltillo y Puebla. Esta experiencia fue seleccionada por presentar características de innovaciones públicas desde abajo, es decir, provenientes de forma autónoma desde la sociedad civil y a la vez hacer uso intensivo de la participación ciudadana por medio de la tecnología basada en Internet, desarrollo de aplicaciones y uso de las redes sociales digitales, por lo que cumple con una buena cantidad de las características de una innovación pública descritas en el marco teórico.

Un caso de estudio es una entidad dotada de límites de espacio temporales, de una estructura y de lógica específica de funcionamiento (Gundermann 2001: 283). En los estudios de caso lo que se pretende es conocer las características y dimensiones que posee el grupo o comunidad estudiado en su particularidad y ver la lógica que relaciona sus elementos y los significados que para sus actores adquieren las interacciones sociales en el particular contexto en que tiene lugar (Gundermann 2001: 260, 283), con el fin de tener una comprensión más profunda del fenómeno bajo estudio en un contexto de vida real (Yin 2004: 1), el cual nos permita responder al planteamiento de un problema, probar o desarrollar una hipótesis o ayudar en la acumulación de conocimiento alrededor de un tema. El caso se analizó a partir de investigación documental, documentos públicos sobre el CIC, sus informes anuales y trabajo de campo de corte cualitativo, consistente 
en la realización de entrevistas a profundidad con Enrique Alanís Dávila y Eduardo Garza Cavazos, Presidente del Consejo y Director General del CIC respectivamente, así como la aplicación de un cuestionario con preguntas abiertas a ocho empleados del centro y el uso de las herramientas creadas por el CIC. Las personas a las que se les aplicó el cuestionario laboran en el CIC en áreas como la dirección general, la dirección de administración y recursos humanos, la coordinación de tecnologías, coordinación de redes sociales, programación, comunicación, psicología y atención ciudadana. Se eligió analizar el caso con una metodología primordialmente cualitativa a fin de obtener información de primera mano que nos ayude a conocer cuáles fueron los factores que convergieron para que surgiera esta innovación pública, así como su funcionamiento y los principales retos que presenta. Al final, se presentan algunas reflexiones a manera de conclusión y se delinean los retos principales que presenta esta experiencia de innovación pública y las limitaciones del modelo de análisis.

\section{PROPUESTA DE MODELO DE ANÁLISIS PARA INNOVACIONES PÚBLICAS}

Para Sørensen y Torfing (2012), la innovación es un proceso dinámico a través del cual los problemas y retos son definidos, nuevas y creativas ideas son desarrolladas y nuevas soluciones son seleccionadas e implementadas. Para Sørensen y Torfing la innovación requiere la activación de emprendedores que articulen problemas, oportunidades y posibles soluciones, así como movilizar recursos materiales y no materiales, para explotar ventanas de oportunidad.

Mientras tanto, la innovación en la esfera pública es definida por Mariñez (2015: 25) como algo más allá de lo meramente gubernamental, implicando compromisos cívicos de colaboración entre funcionarios, sector privado, ciudadanos y organizaciones de la sociedad civil, que permiten producir cambios en valores, actitudes, procesos y liderazgos que contribuyan con nuevas soluciones a las demandas sociales, generando con ello valor público. En ese mismo sentido, Zubbrigen y González (2014: 331) señalan que el surgimiento de formas innovadoras de participación responde a un modelo de gobernanza colaborativa, en las que ciudadanos y comunidades desempeñan un papel activo en la co-creación de servicios y políticas públicas, a la vez que las nuevas tecnologías de la información habilitan nuevas formas de participación.

Si bien el concepto general de innovación planteado por Sørensen y Torfing (2012) nos plantea la importancia de nuevas soluciones y la activación de emprendedores que las lleven a cabo, Mariñez (2015), 
Zubbrigen y González (2014) se adentran en la aplicación de este concepto en el ámbito público, el cual va más allá de la esfera del gobierno e incluye una multiplicidad de actores públicos para detonar y desarrollar esta innovación, los cuales a su vez están envueltos en dinámicas propias y complejas, que hacen necesarios esquemas de colaboración flexibles, los cuales pueden ser mediados y facilitados por la tecnología.

Ninguno de los autores reseñados dice nada acerca de cómo surge y quién lidera el proceso de innovación pública, cuestión que es abordada por Kallio, Lappalainen y Tammela (2013), quienes señalan que la participación de los ciudadanos impacta positivamente en la capacidad de innovar de las organizaciones públicas y puede ser un acelerador de los procesos de innovación y una fuente de ideas nuevas y radicales, en los que los innovadores ya no son tan solo los funcionarios públicos, sino los usuarios ciudadanos en si mismos. Para Kallio, Lappalainen y Tammela, el proceso de innovación puede darse de forma planeada, en donde el gobierno involucra a los ciudadanos, en un esquema tipo topdown, o de arriba hacia abajo, que tiende a ser lento, pero involucra un proceso de aprendizaje entre funcionarios y ciudadanos en un proceso de co-innovación, o bien de tipo bottom-up, o de abajo hacia arriba, en el cual ciudadanos involucrados en cuestiones sociales actúan de forma empoderada para brindar soluciones y servicios de manera autónoma o sin una presencia directa de actores gubernamentales, en un proceso de innovación acelerado liderado por ciudadanos, del cual se apropian y que sirve de aprendizaje al gobierno, innovando en áreas o servicios que les afectan directamente o que son de su interés.

Con las nuevas tecnologías basadas en Internet, tales como redes sociales, blogs, wikis, canales en Youtube y aplicaciones (apps), los esquemas de innovación pública liderados por los ciudadanos se han multiplicado. Sin embargo, autores como Bäck et al. (2013) señalan que es importante ir más allá de la simple discusión en redes sociales sobre los problemas públicos. En cambio, la innovación pública puede detonarse mediante esquemas que liguen dichas tecnologías de la información con datos abiertos del gobierno y permitan detectar problemas y diseñar soluciones conjuntas. Para García (2014), por datos abiertos se entienden aquellos que los gobiernos recopilan para sus propias funciones y que luego liberan en formatos modificables para ser reutilizados y redistribuidos por ciudadanos y organizaciones para diversos fines.

En ese sentido, Rojas-Martín y Criado (2015) señalan que el creciente uso de redes sociales digitales y otras tecnologías enfocadas a resolver problemas sociales pueden potenciar la relación entre el gobierno y la sociedad para 
lograr la co-creación de servicios y hacer una toma de decisiones más inclusiva por medio de comunidades colaborativas virtuales, transitando así del gobierno electrónico unidireccional al llamado Gobierno 2.0, el cual además de permitir una comunicación bidireccional permite la cocreación.

Los esquemas de innovación pública pueden aprovechar el creciente uso del crowd sourcing, o fuentes de información masivas, entendidas como una

[...] actividad en línea participativa en la que una persona, institución, organización de la sociedad civil o empresa propone a un grupo de individuos mediante una convocatoria pública flexible la realización libre y voluntaria de una tarea de complejidad variable y en que la multitud debe participar aportando su conocimiento, experiencia, trabajo o dinero, mientras que el usuario recibirá la satisfacción de una necesidad concreta, ya sea económica, de reconocimiento social, auto estima o desarrollo de aptitudes personales, mientras que el crowdsourcer obtendrá y utilizará en beneficio la aportación del usuario (Estellés y González, citados en Pardo 2014).

Algunos de los tipos más utilizados de crowd sourcing tienen que ver con el crowd funding o financiamiento masivo, el cual permite obtener grandes cantidades de recursos de pequeñas aportaciones realizadas por personas a través del mundo, ligando aplicaciones de teléfonos inteligentes, tablets y computadoras con sistemas de pago bancarios en línea, tarjetas de crédito o servicios como Pay Pal. Estas aplicaciones permiten hacer frente a necesidades de financiamiento de una manera más rápida que las formas tradicionales de recaudación de fondos. Un ejemplo exitoso es la aplicación ShareTheMeal.org, la cual fue desarrollada por un grupo pequeño de 14 jóvenes voluntarios en Alemania para el Programa Mundial de Alimentos de las Naciones Unidas, mediante la cual se han logrado recaudar fondos en pocas semanas para proyectos tales como alimentar todo un año a niños refugiados sirios en campamentos en Jordania y madres embarazadas en etapa de lactancia y sus bebes en ciudades sirias como Homs, destrozadas por los bombardeos (www.sharethemeal.org).

Otro tipo de crowd sourcing desarrollado tanto por gobiernos como organizaciones de la sociedad civil es el crowd creation o creación masiva, la cual involucra la resolución de tareas, retos o problemas que requieren habilidades creativas o innovadoras por parte del público (Pardo 2014). Las creaciones masivas pueden ser iniciativas tanto del gobierno como de las organizaciones de la sociedad civil y se basan en el entendimiento de que entre más opiniones e ideas diversas se tomen en cuenta más integral 
será la solución propuesta para hacer frente a una necesidad, fenómeno o problema. Estas co-creaciones pueden ser divididas en micro tareas o micro proyectos, así especialistas en cada una de las etapas de los mismos pueden aportar conocimiento de gran calidad para la solución propuesta.

Los esquemas de crowd sourcing involucran el uso de datos abiertos, una gran participación cívica o ciudadana y esquemas de colaboración entre el gobierno, las organizaciones de la sociedad civil y la iniciativa privada. Para Bäck et al. (2013), un requerimiento para impulsar la participación cívica es que sus alcances deliberativos y formas de involucramiento sean accesibles para la mayor cantidad de personas.

En el crowd sourcing las tecnologías basadas en Internet permiten procesos catalogados como produsage o de producción-uso (Bruns, citado por Bäck et al. 2013), en los cuales los usuarios ciudadanos están continuamente usando y produciendo servicios, como sucede en Wikipedia, bajo los siguientes principios: 1) participación abierta y evaluación comunal; 2) jerarquía fluida con meritocracia $a d-h o c$; 3) instrumentos sin terminar y en continuo proceso; y 4) propiedad común con recompensas individuales.

Otro tipo de crowd sourcing es el crowd voting o votaciones masivas, las cuales según Pardo (2014), son votaciones por medio de Internet para organizar, filtrar y calificar contenidos. Para Surowiecki, citado por Bäck et al. (2013) estos esquemas de crowd sourcing se basan en el principio del crowd wisdom o sabiduría de las masas, en el cual grupos grandes de personas pueden producir predicciones y decisiones colectivas más inteligentes que pocos expertos, siempre y cuando existan condiciones tales como diversidad, independencia, descentralización de opiniones y una forma de agregar opiniones en una sola respuesta, por medio de metodologías como el crowd storming o tormenta de ideas masiva. Esta sabiduría de masas evita en palabras de García (2014: 76) la pérdida del acervo de conocimientos, habilidades y creatividad disponibles en el conjunto de la sociedad, lo que indica, supondría una ineficiente gestión de los recursos disponibles.

Para Surowiecki y Oinas-Kukkonen, citados por García (2014: 83), para que los datos abiertos y la sabiduría de masas produzcan resultados positivos son necesarios los siguientes criterios:

- Oportunidad. La información ha de llegar en el momento adecuado, al grupo adecuado, por el canal adecuado.

- Diversidad de opinión. Cada individuo debe formar un juicio propio sobre la problemática en estudio.

- Independencia. Las opiniones de los individuos no deben verse 
afectadas por las opiniones del resto.

- Competencia. Los resultados óptimos nacerán de permitir desacuerdo y rivalidad entre ideas.

- Descentralización. Los datos han de permitir que los individuos enfoquen su actuación sobre problemáticas locales;

- Economicidad. Un exceso de comunicación generará costes de transacción que reducirán el rendimiento del grupo;

- Agregación. Han de existir las herramientas que permitan convertir las opiniones y propuestas privadas en acciones colectivas.

La interacción colaborativa, según Sørensen y Torfing (2012), facilita una circulación basada en la confianza y una fertilización cruzada de nuevas ideas. De esta manera, la colaboración es muy importante en la innovación pública y a su vez impacta en los procesos de política pública, no solo legitimando las decisiones y haciéndolas más inclusivas, sino redefiniendo las problemáticas de manera conjunta, co-creando soluciones, implementándolas y evaluándolas.

En ese sentido, Bäck et al. (2013) señalan que los servicios basados en redes sociales digitales deben ser diseñados para apoyar las acciones colaborativas y la comunicación, lo cual es abordado también por García (2014: 75), quien señala que la tecnología y la cultura digital que se ha instaurado en la sociedad han creado un mundo más transparente y procesos de creación más participativos y colaborativos, introduciendo un elemento disruptivo del proceso democrático al renovar el interés ciudadano por lo público y en el cual las tecnologías de la información basadas en Internet han posibilitado el mayor nivel de comunicación participativa global e intercultural que la humanidad haya conocido, quitándole a los funcionarios y especialistas el monopolio del conocimiento. Lo anterior, coincide con los señalado por Santiago (2014), que señala que la innovación fomenta procesos de desarrollo más inclusivos y participativos, beneficiándose de la variedad de actores participantes, dando espacio a la convergencia de más oportunidad y más participación en la toma de decisiones de todos los actores involucrados.

A partir de los autores analizados, particularmente de Kallio, Lappalainen y Tammela (2013), Zubbrigen y González (2014), así como Mariñez (2015), encontramos que en la innovación pública más allá de lo que pueda hacer diferente el gobierno, lo cual es considerado propiamente como innovación gubernamental. Es muy importante la participación de la ciudadanía, tanto organizada como a nivel individual, para lo cual se requieren mecanismos de organización, agregación y jerarquización para participar de manera conjunta con otros actores en la definición de las 
problemáticas, el diseño de soluciones, su implementación y evaluación. Adicionalmente, el uso de la tecnología, aunque no es lo que define en sí a la innovación, si la potencia, sobre todo lo referente a las redes sociales digitales, el desarrollo de aplicaciones propias (apps) y otras tecnologías basadas en Internet, como señalan Bäck et al. (2013), García (2014), así como Rojas-Martín y Criado (2015), las cuales son utilizadas para facilitar la participación ciudadana, así como aprovechar la información disponible en forma de datos abiertos o bien para crear su propio conjunto de información con la participación de las masas, que sirva para identificar problemas públicos, movilizar recursos y generar soluciones innovadoras que permitan su solución.

Así, para efectos de este artículo, proponemos definir la innovación pública como aquel proceso liderado por la sociedad civil, que en conjunto con otros actores como el gobierno y empresas utiliza la sabiduría colectiva para la detección conjunta de necesidades públicas, la creación e implementación de soluciones masivas creativas, haciendo uso intensivo de redes sociales digitales y otras tecnologías basadas en Internet.

Con esta discusión teórica como base, se propone un modelo de análisis de experiencias de innovación pública, por medio de la construcción de categorías taxonómicas, las cuales cómo señala Sartori (2011) se refieren a clasificaciones o contenedores de datos conceptualmente definidos, que permiten analizar un fenómeno que reúne similares características, es decir, que es de la misma clase y diferenciarlo de los que tienen características diferentes. 


\section{Tabla 1: Modelo para el análisis de experiencias de innovación pública \\ Categorías \\ Variables}

Tipo de

surgimiento y

desarrollo

Participación de la ciudadanía

Tipo de organización

Etapa de evolución

Tipo de actores con los que colaboran

Tipo de tecnología

Tipos de crowd sourcing

Tipo de datos creados y utilizados

Nivel de transparencia

Posibles impactos sociales
Top-down. La innovación surge desde el gobierno,

Bottom-up. La innovación surge desde la sociedad civil.

Híbrido. La innovación surge de manera conjunta entre sociedad civil y gobierno.

Solo por medio de sociedad civil organizada

Solo de forma individual

Mixta

Voluntaria

Institucionalizada

Nueva creación

En maduración

Madura

Organizaciones de la sociedad civil

Gobiernos

Empresas

Uso intensivo de redes sociales digitales como centro de la innovación.

Uso intensivo de otras tecnologías basadas en Internet como centro de la innovación.

Unidireccionales. La comunicación fluye principalmente desde el actor que genera la innovación hacia otro.

Bidireccionales. La comunicación fluye de manera equilibrada desde el actor que genera la innovación hacia otro y viceversa.

Multidireccionales. La comunicación fluye de manera equilibrada desde el actor que genera la innovación hacia otros y viceversa.

\section{Crowd wisdom}

Crowd creation

Crowd funding

Crowd voting

Uso de datos abiertos creados por el gobierno

Generación y uso de datos abiertos por parte de las organizaciones de la sociedad civil.

Transparencia financiera, de procesos y resultados (baja, media, alta).

Fortalecimiento del tejido social, mayor participación ciudadana, colaboración entre actores, mejora de transparencia, rendición de cuentas, impacto sobre las condiciones de vida de la ciudadanía.

Fuente: Elaboración propia. Algunas variables introducidas han sido analizadas por Kallio, Lappalainen y Tammela (2013), Bäck et al. (2013), Mariñez (2015), Rojas-Martín y Criado (2015). 
Así, el caso del CIC es analizado a la luz de estas categorías, compuestas a su vez de variables. Se proponen diez categorías relativas al tipo de surgimiento y desarrollo, tipo de participación de la ciudadanía, tipo de organización, etapa de evolución, los actores con los que colaboran, tecnología utilizada, tipos de crowd sourcing, tipo de datos creados y utilizados, nivel de transparencia y posibles impactos sociales generados. Cada una de esas categorías y variables permiten a su vez interpretar un nivel de graduación de dichas intensidades. A continuación describiremos el caso del CIC de acuerdo a la investigación documental y el trabajo de campo realizado, para posteriormente analizar la experiencia del CIC utilizando este modelo

\section{ANÁLISIS DE CASO: EL CENTRO DE INTEGRACIÓN CIUDADANA}

El CIC es una organización de la sociedad civil que tiene su base en San Pedro Garza García, Nuevo León, México y cuenta con una herramienta llamada Tehuan, que significa "nosotros" en lengua náhuatl, la cual que enlaza a los ciudadanos y las autoridades, al recibir, validar, canalizar y dar seguimiento a los reportes ciudadanos recibidos por medio de Twiter, aplicaciones móviles, su página de Internet, un teléfono 01800 y servicios de mensaje de texto (SMS), publicando los reportes de forma georreferenciada en temas como emergencias, seguridad pública, vialidad y tránsito, así como servicios públicos, en toda el Área Metropolitana de Monterrey, así como las ciudades de Saltillo y Puebla (http://www.cic.mx).

Cuando nace el CIC el objetivo primordial era que ante la situación de inseguridad muy poca gente denunciaba, por desconfianza, por la complejidad que tiene el proceso de denuncia o el miedo a represalias. Nosotros surgimos como un enlace a través de la tecnología para facilitar los reportes ciudadanos y si alguien quiere denunciar, nosotros aquí los arropamos para que no se sientan solos durante el proceso para explicarles cómo se hace una denuncia, inclusive tenemos muy buenas relaciones con gobiernos donde también ellos vienen aquí a la oficina a levantar denuncias si es necesario (Garza Cavazos, Eduardo. Entrevista, 31 de mayo del 2016).

En el año 2015 se atendieron 15.980 reportes referentes a vialidad, como choques y autos descompuestos, 9.096 reportes de servicios públicos como alumbrado público, alcantarillas, semáforos descompuestos, faltas de electricidad, baches, fugas, parques descuidados y recolección de basura y 1,449 reportes sobre temas de seguridad, tales como secuestro, robos, extorsión, homicidio, persona sospechosa, auto abandonado, detención de 
bandas criminales, percepción de inseguridad o situaciones de riesgo (CIC 2015).

En el marco de la inseguridad vivida en México, las situaciones de riesgo son entendidas como aquellos eventos que ponen en riesgo la vida, la integridad física y/o psicológica de los ciudadanos, tales como: balaceras; granadazos; poncha llantas y retenes ilegales por parte de la delincuencia organizada utilizados para robar o secuestrar; o bien los narco-bloqueos viales que representan el modus operandi de la delincuencia para evitar el traslado y llegada de la Policía, la Marina o el Ejército, en los que roban a mano armada camiones o vehículos pesados, los incendian y atraviesan en las carreteras o avenidas para detener la circulación. El hashtag o etiqueta \#SDR es ampliamente utilizado a diario en redes sociales como Twitter y Facebook en ciudades como Monterrey, Nuevo Laredo, Ciudad Victoria, Reynosa, Guadalajara, Morelia, para alertar sobre estos eventos a la población. Incluso, algunos twitteros han sido asesinados por parte de la delincuencia organizada por dar estas advertencias, como el caso de la Dra. María del Rosario Fuentes Rubio, conocida en Twitter como @Miut3, secuestrada y asesinada en Reynosa en el $2014^{1}$, de ahí la importancia de este tipo de herramientas del CIC, que dan oportunidad del anonimato a la población.

Respecto de los reportes por cuestiones de seguridad, Eduardo Garza Cavazos, señaló que cada vez llegan con mayor detalle, ya que reportan el número de placa de los autos involucrados y el color, lo que ayuda a las autoridades, ya que anteriormente se recibían reportes escuetos (Entrevista, 31 de mayo del 2016).

Además de dar seguimiento a los reportes enviados a las autoridades por medio de esta herramienta tecnológica, el CIC brinda asesoría legal y psicológica a víctimas de delitos. Durante el 2015 se brindaron 1.071 acciones de asesoría jurídica a 431 personas, iniciando 89 casos legales, de los cuales resultaron 23 denuncias interpuestas, en coordinación con Fuerza Civil, por medio de la cual los ciudadanos pudieron interponer denuncias directamente en las oficinas del CIC, siendo en este caso el delito de extorsión el atendido con mayor frecuencia (CIC 2015: 4-5).

En el acompañamiento legal "estamos muy enfocados en delitos de alto impacto, como violación, secuestro y asesinato, son los principales

1 Véase http://www.multimedios.com/telediario/en-alerta/hoy-mi-vida-llegadotuitera.html y http://aristeguinoticias.com/1710/mexico/difunden-amenazas-a-latuitera-asesinada-en-tamaulipas/ 
delitos en los que brindamos apoyo para denunciar. También en robo con violencia entramos a apoyar en la denuncia" (Garza Cavazos, Eduardo.. Entrevista, 31 de mayo del 2016).

Sobre la asesoría psicológica, se brindó orientación, asesoría y tratamiento a un total de 2.298 personas durante el mismo año, de las cuales 51 casos se derivaron de un delito, como violencia familiar, agresión sexual y secuestro. El 61\% de las asesorías psicológicas se dieron por medio del chat (CIC 2015: 5).

Por teléfono o chat por ejemplo nos llegan problemas de suicidio, en estos casos primero se interviene en la crisis, se trata de calmar a las personas que están en riesgo y después se les asesora con quien pueden ir para que reciban tratamiento en este tema y si es necesario se les vincula a una organización cercana a ellos, porque nos llegan casos de todo el país (Garza Cavazos, Eduardo. Entrevista, 31 de mayo del 2016).

Muchas gracias por todo, todos estos meses, siento que volví a nacer con su ayuda, a creer y confiar en el rumbo que quiero para mi vida. Se ganaron un lugar en mi corazón y deseo que sigan creciendo y ofreciendo todo el apoyo a las personas (Anónimo. Víctima de violación e intento de suicidio atendida. CIC 2012: 10).

Según el cuestionario aplicado al equipo del CIC, estos consideran que los esfuerzos que realizan están relacionados con la innovación pública, cívica y tecnológica, así como con esquemas de gobierno abierto y que el impacto que han logrado en la sociedad desde el centro está relacionado principalmente con:

- Empoderar a los ciudadanos, al tomarlos en cuenta y atender de forma directa sus necesidades por medio de sus reportes;

- Unir comunidades y ser un puente real para entre la sociedad y las autoridades.

- Brindar asesoría legal y apoyo psicológico a víctimas de delitos de alto impacto.

- Ser innovador en el uso de tecnologías para que los ciudadanos puedan involucrarse en su comunidad y generar datos confiables y abiertos sobre incidencia delictiva que sirvan para que los gobiernos puedan mejorar su procesos.

- Devolver el bienestar social y personal que se había perdido hace unos años. 
A continuación, analizamos el CIC con base en el modelo presentado en la Tabla 1, para conocer qué características tiene a la luz de las principales teorías sobre innovación pública.

Respecto al tipo de surgimiento o desarrollo, este tipo de innovación pública es un claro ejemplo del esquema bottom-up o de abajo hacia arriba, de acuerdo con las categorías planteadas por Kallio, Lappalainen y Tammela (2013), permitiendo un empoderamiento ciudadano acelerado, gracias al cual el gobierno recibe información de calidad, generada por las masas y organizada por la sociedad civil con la ayuda de las tecnologías de la información, para la detección y solución de problemas públicos.

La participación de la ciudadanía en este tipo de innovación pública es mixta, pues si bien su surgimiento se da por parte de la sociedad civil organizada, se requiere de una alta participación ciudadana en lo individual, pues son los ciudadanos los que generan los reportes. Sobre el tipo de organización, esta es institucionalizada, es decir, está conformada como una asociación civil registrada ante notario público y está gobernada por un consejo directivo y un consejo consultivo ciudadano que se reúnen periódicamente para revisar los avances y realizar propuestas de mejora (http://www.cic.mx/preguntas-frecuentes/). Sobre las motivaciones para formar parte del CIC, los empleados a los que se les aplicó el cuestionario mencionaron en su mayoría cuestiones altruistas y de superación personal, así como motivaciones salariales, lo cual es un indicador de que los sueldos ofrecidos permiten atraer talento y pasar de un trabajo voluntario a uno pagado.

En estos momentos trabajando en el CIC tenemos 32 personas: en el área de tecnología tenemos a cuatro personas; en el área de psicología tenemos a tres personas aquí fijas en oficinas y cinco personas afuera dando apoyo psicológico en crisis con empresas colaboradoras; en apoyo jurídico tenemos a tres personas; en comunicaciones tenemos a siete personas, que son diseñadores y los que están enfocados en la capa de análisis del Twitter y Tehuan; en administración son dos personas, un contador y una asistente administrativa; en la recepción otra persona; el director; los demás son practicantes. En Saltillo abrieron con siete personas y en Puebla con cuatro (Garza Cavazos, Eduardo. Entrevista, 31 de mayo del 2016).

Es una organización de la sociedad civil en etapa de maduración, pues como menciona Enrique Alanís (Entrevista, 14 de junio del 2016), el proyecto nació como idea en el año 2010 en medio de la ola de delincuencia que azotó el país y particularmente a Nuevo León, iniciando operaciones 
formalmente en octubre del 2011 (CIC 2012), es decir, tiene cinco años de existir a momento de publicar esta investigación (enero del 2017). Según el cuestionario aplicado al equipo del CIC, en promedio a la misma fecha los empleados del CIC llevan trabajando en la organización tres ańos y cuatro meses, lo que nos habla de que existe una baja rotación laboral, por lo puede darse una experiencia acumulada de los procesos en la organización y una mayor especialización en el trabajo. En referencia a la figura del presidente del consejo, han existido cuatro personas en total incluyendo al actual. Además del Área Metropolitana de Monterrey, el CIC opera ahora también desde el 2013 en la ciudad de Saltillo (CIC 2013) y desde el 2014 en Puebla (CIC 2014).

Con referencia a los tipos de actores con los que colaboran, el CIC colabora con la sociedad en general por medio de la recepción de los reportes y la atención a víctimas, además del gobierno para canalizar dichos reportes, con otras organizaciones de la sociedad civil que buscan objetivos similares y empresas que apoyan su financiamiento. "Colaboramos en varios programas cívicos, como Consejo Cívico estamos en "Nuevo León ¿Cómo vamos?”, "Alcalde ¿Cómo vamos?”, con Renace, Hagámoslo Bien, Arval, Pazes y Codeando México" (Garza Cavazos, Eduardo. Entrevista, 31 de mayo del 2016).

Los integrantes del equipo de trabajo del CIC consultados consideran que si bien ha costado trabajo, la relación con las autoridades municipales y de seguridad ha sido buena y constante, contando con convenios formales de colaboración con la mayoría de las ciudades del Área Metropolitana de Monterrey y siendo tomadas en cuenta la mayoría de sus iniciativas. Mencionaron que aunque ha habido apertura, en algunos casos los funcionarios ven la labor del centro como una competencia o intrusión hacia su trabajo, con miedo a transparentar su trabajo. Adicionalmente, los cambios de administración hacen necesarios acercamientos con los nuevos alcaldes y gobernadores.

Cada vez llega alguien con una nueva mentalidad y este es un reto contante, volver a hablar de los sistemas, de los beneficios, atiéndeme, atiende al ciudadano. Todo eso de estar vinculando al gobierno es un reto constante, porque también te pueden cerrar las puertas sin ningún problema (Garza Cavazos, Eduardo. Entrevista, 31 de mayo del 2016).

La tecnología ha sido básica para cumplir con los objetivos del CIC por medio de su principal herramienta, Tehuan, la plataforma tecnológica que recibe y difunde reportes de hechos observados por los ciudadanos 
vía Twitter, correo electrónico, App móvil, mensajes de texto por teléfono celular (SMS) o página de Internet. Los reportes pueden hacerse incluso vía anónima, por ejemplo, en situaciones de riesgo relacionados con el crimen organizado donde se puedan temer represalias. En los reportes por medio de la App se pueden compartir fotos de lo que se reporta y se cuenta con georreferenciación. Además del seguimiento que hace el CIC de cada uno de los reportes con las autoridades correspondientes, las mismas tienen acceso directo a los mismos para atender situaciones urgentes. Se hace así un uso intensivo de redes sociales digitales y otras tecnologías basadas en Internet con un uso multidireccional de las mismas. Del total de los 19.649 reportes realizados en el 2015 , el $70 \%$ se hicieron a través de Twitter, $18 \%$ por la página de Internet del centro, $7 \%$ por medio de la App, 3\% por Whatsapp, que se comenzó a utilizar en octubre de ese año y el 2\% por correo electrónico (CIC 2015: 16).

El uso de plataformas tecnológicas para ser utilizadas en teléfonos inteligentes ha sido una de las apuestas del centro, lo que ha sido posible gracias al hecho de que tan solo en el 2015 la tasa de crecimiento del uso de estos aparatos creció 43,2\% en México, por lo que 7 de cada 10 celulares utilizados en el país son de este tipo (The Competitive Intelligence Unit 2015, citada en CIC 2015). En el caso de la cuenta de Twitter @CICMty tiene 103.713 seguidores, mientras que su página de Facebook tiene 67.771 likes y la App en total ha sido descargada 13.530 veces (CIC 2015).

Entre los principales logros del CIC está el haber ganado en el año 2014 el premio con financiamiento New Digital Age Grants, fundado a través de un donativo de US\$1.000.000 por Eric Schmidt, ex director de Google y que fue otorgado a diez organizaciones en total que utilizan tecnología para la resolución de los problemas más graves y urgentes que enfrenta la sociedad (http://www.cic.mx/google-executive-chairman-eric-schmidtawards-centro-de-integracion-ciudadana-a-new-digital-age-grant/). También logró ganar en el 2015 el tercer lugar del premio a Proyectos Tecnológicos para la Prevención de la Violencia de Motorola Foundation (CIC 2015). Según el equipo del CIC, entre sus retos está continuar innovando en sus plataformas tecnológicas y seguir con la capacitación constante en nuevas herramientas que permitan tener una operación más esbelta y reducir costos.

Mi reto es incrementar, desarrollar e inventar nuevas cosas. Uno de los valores que hemos tenido en el CIC es la innovación. El propósito del CIC es crear tecnologías de información que empoderen al ciudadano y que al mismo tiempo faciliten el trabajo de las autoridades (Alanís, Enrique. Entrevista. 14 de junio del 2016). 
Sobre los tipos de crowd sourcing que son utilizados por el CIC, consideramos que el principal es uso del crowd wisdom, ya que al permitir que los ciudadanos hagan sus reportes directamente por medio de la App y explicar que es lo que está pasando, acompañando sus reportes sobre situaciones de riesgo o de servicios con fotos y georreferenciación, se aprovecha el conocimiento que ellos tienen de su entorno para su posterior evaluación y atención. También tienen una acción denominada Perspectiva Ciudadana, que consta de un espacio para que los ciudadanos compartan propuestas de ideas innovadoras con el CIC y el público en general, promoviendo así un intercambio de ideas que enriquezcan futuras acciones ciudadanas (http://www.cic.mx/perspectiva-ciudadana).

El crowd wisdom también es utilizado para saber más sobre situaciones de riesgo en proceso.

Hay muchas experiencias donde la misma autoridad nos pide más información. Le llega un reporte y quieren conocer más, si tenemos información sobre determinado reporte, que le preguntemos a nuestras redes ciudadanas para saber que está pasando y eso ha pasado con la Fuerza Civil y con el Ejército (Alanís, Enrique. Entrevista. 14 de junio del 2016).

Sobre el crowd funding, el CIC financia sus actividades por medio de donatarios corporativos y de manera incipiente mediante esquemas de financiamiento masivo usando herramientas como Pay Pal.

Nos falta mucho para que se genere una cultura así (de crowd funding). Es más institucional nuestro financiamiento, aunque también existen donativos por parte de los beneficiados que atendimos en temas psicológicos y legales, gente agradecida que ofrece donativos. Falta crear ese círculo virtuoso, de decir: yo doy para esta causa pues beneficia a todos y quiero dar (Alanís, Enrique. Entrevista. 14 de junio del 2016).

El total de donativos al CIC durante el 2015 ascendió a MX \$ 15.628.137 pesos (US \$ 919.302, a un tipo de cambio de 17 pesos por dólar), de los cuales el $88,6 \%$ provino de empresas y el resto de los premios ganados mencionados anteriormente (CIC 2015). Según Eduardo Garza Cavazos, las empresas que aportan económicamente y en especie son muchas, pero es Cementos Mexicanos (CEMEX) el principal donatario, que a su vez es la empresa que ideó la creación del CIC en el 2010, bajo el liderazgo de Lorenzo Zambrano (RIP), entonces presidente de la compañía (Entrevista, 31 de mayo del 2016). 
Según el equipo del CIC consultado por medio del cuestionario, los principales desafíos que enfrenta el centro a futuro tienen que ver con tener un mayor presupuesto, a partir de lograr donativos de empresas con las que colaborar y tener así la posibilidad de contratar más personal, para poder cumplir con sus metas. Otro reto señalado por Eduardo Garza Cavazos, tiene que ver con el crecimiento a más ciudades del país, lo que implica que empresas y organizaciones de la sociedad civil locales se apropien y financien el proyecto y hagan vínculos con el gobierno local, pero sin recibir fondos del mismo, lo cual no se permite por políticas internas del CIC (Entrevista, 31 de mayo del 2016).

Al momento de la presente investigación no se documentó el uso de estrategias del tipo crowd creation o crowd voting por parte del CIC. Sobre el tipo de datos creados y utilizados, son los propios ciudadanos lo que generan los datos, que el CIC georreferencía, organiza, analiza y comparte en forma de datos abiertos no solo para las autoridades, sino para el público en general, para que la pueda usar con múltiples propósitos, como saber las zonas donde existe mayor incidencia delictiva o de falta de servicios, a fin de crear estrategias de prevención y atención.

Siguiendo con el modelo de análisis, el nivel de transparencia financiera, de procesos y resultados del CIC es alto. Publican constantemente información sobre esto en su página de Internet, además de informes anuales, cuyos resultados financieros son auditados por un despacho contable.

Por último, respecto al impacto social del CIC, este ha elevado la participación ciudadana, la colaboración entre actores y ha coadyuvado para que diferentes instancias gubernamentales den seguimiento y solución a distintas problemáticas sociales, lo que en conjunto ha abonado para fortalecer el tejido social. Dado el uso de la tecnología para el levantamiento, validación, clasificación, canalización, seguimiento y georreferenciación de los reportes, la plataforma del CIC genera datos abiertos para múltiples usos por parte de las autoridades y los ciudadanos en general. Además, ha servido de acompañamiento legal y psicológico a las víctimas de delitos de alto impacto, lo que se traduce en una mejora de las condiciones de las mismas en hechos tan traumáticos como un delito que atente contra su integridad.

\section{CONCLUSIONES}

La innovación pública se detona por medio de esquemas liderados por organizaciones de la sociedad civil, donde participan activa y directamente 
ciudadanos, que colaboran con diversas instancias gubernamentales para la creación de un mayor valor público. El CIC es una organización de la sociedad civil institucionalizada y en etapa de maduración, cuyas actividades cumplen con la definición de innovación pública propuesta en el presente trabajo y por autores como Mariñez (2015), así como Zubbrigen y González (2014), siendo sus componentes principales: la participación ciudadana organizada en torno a un emprendimiento social, la participación ciudadana masiva, la colaboración con el gobierno y el uso de las tecnologías de la información basadas en Internet con uso intensivo de redes sociales digitales.

Se encontró que el CIC enlaza exitosamente a ciudadanos y autoridades, al recibir, validar, clasificar, canalizar y dar seguimiento a los reportes de la población. Además de dar seguimiento a los reportes canalizados a las autoridades, brinda asesoría legal y psicológica a víctimas de delitos, lo que concuerda con lo planteado por Kallio, Lappalainen y Tammela (2013), Zubbrigen y González (2014), así como Mariñez (2015), quienes ven la participación ciudadana directa como indispensable para que existan esquemas de auténtica innovación pública, colaborando en el caso del CIC con distintos órdenes de gobierno para poder tener un impacto real en la solución de los problemas públicos.

EI CIC utiliza intensivamente sus propias aplicaciones tecnológicas (apps), redes sociales digitales, y otras herramientas basadas en Internet, así como telefonía fija y móvil, publicando los reportes de forma georreferenciada en temas como situaciones de riesgo, emergencias, seguridad pública, vialidad y tránsito, así como servicios públicos. El uso de estas tecnologías según Bäck et al. (2013), García (2014), así como Rojas-Martín y Criado (2015), permiten potenciar y hacer más ágiles los procesos de innovación pública, siendo en el caso del CIC una de sus principales fortalezas, pues desde su origen ha impulsado la creación y uso de tecnologías creativas para la re solución de problemas públicos, además de estar presente su mejora constante.

Si bien el CIC cuenta con un presupuesto considerable, entre sus retos está el diversificar sus principales fuentes de ingresos, para que no dependan tanto de una sola empresa, CEMEX, haciendo uso intensivo de esquemas de crowd funding que se enlacen con sus principales plataformas tecnológicas, así como de campañas financieras nacionales e internacionales más agresivas. Lo anterior, para poder mantener su proceso de innovación en plataformas tecnológicas y contar con compensaciones competitivas para su personal de base, mantener una baja rotación laboral y seguir atrayendo talento. 
Relacionado con lo anterior, otro reto tiene que ver con su expansión geográfica, que depende tanto de la existencia de una sociedad civil organizada y una clase empresarial local dispuesta a invertir en las plataformas y recursos humanos, como de la voluntad política de los gobiernos para colaborar. En este sentido, es necesaria una mayor disponibilidad presupuestal, tanto para financiar programas piloto, como para demostrar el uso de la herramienta en potenciales ciudades interesadas, sobre todo aquellas donde se da una combinación entre altos niveles de violencia, baja tasa de denuncias, déficit de sociedad civil organizada y baja capacidad institucional de las autoridades, pues si bien el Área Metropolitana de Monterrey tuvo niveles altos de violencia y fue en dónde nació este esfuerzo, la presencia de empresarios con recursos, sociedad civil organizada, ciudadanos comprometidos y gobiernos dispuestos a colaborar no es común en el resto de la República Mexicana.

El modelo de análisis propuesto en la presente investigación permitió profundizar en el caso del CIC y conocer a detalle esta experiencia de innovación pública. Si bien se procuró que el modelo fuera exhaustivo en la propuesta de categorías y variables analíticas, es solo un punto de partida para formalizar el análisis de este tipo de experiencias de innovación pública, que deberá ser refinado y enriquecido, a partir de su calibración en otros casos similares. 


\section{REFERENCIAS}

Bäck, A., Friedrich, P., Ropponen, T., Harju, A. y Hintikka, K. A. (2013). From Design Participation to Civic Participation - Participatory Design of a Social Media Service. International Journal of Social and Humanistic Computing, 2 (1-2), 51-67.

Centro de Integración Ciudadana (CIC) (2012). Informe Anual de Actividades. Monterrey: Centro de Integración Ciudadana. Disponible en: https://storage.googleapis.com/cic-icalia/InformeAnual2012.pdf [01-06-2017].

. (2013). Informe Anual de Actividades. Monterrey: Centro de Integración Ciudadana. Disponible en: https://storage.googleapis. com/cic-icalia/CIC_2014_final.pdf [01-06-2017].

. (2014). Informe Anual de Actividades. Monterrey: Centro de Integración Ciudadana. Disponible en: http://cic-icalia.storage. googleapis.com/CIC_DIGITAL-3.pdf [01-06-2017].

. (2015). Informe Anual de Actividades. Monterrey: Centro de Integración Ciudadana. Disponible en: http://cic-icalia.storage. googleapis.com/InformeAnual2015.pdf [01-06-2017].

García, J. (2014). Gobierno Abierto, Transparencia, Participación y Colaboración en las Administraciones Públicas. Innovar. Revista de Ciencias Administrativas y Sociales, 24 (54), 75-88.

Gundermann, H. (2001). El Método de los Estudios de Caso. En Tarres, M. (coord.), Observar, Escuchar y Comprender. Sobre la Tradición Cualitativa en la Investigación Social. México: Miguel Ángel Porrúa, Flacso.

Kallio, K., Lappalainen, I. y Tammela, K. (2013). Co-innovation in Public Services: Planning or Experimenting with Users? The Innovation Journal. The Public Sector Innovation Journal, 18 (3), 1-16.

Mariñez, F. (2015). Innovación Pública. Para Que Funcionarios Públicos y Ciudadanos Actúen con Saberes Cívicos. México: Editorial Fontamara.

Pardo G. (2014). Crowdsourcing en Procesos de Gobierno Abierto. Valencia: Universitat Politècnica de València. 
Rojas-Martín, F. y Criado, J. (2015). Avanzando en la Medición de las redes Sociales Digitales: Una Propuesta para su Análisis desde una Perspectiva Institucionalista. Revista de Gestión Pública, 4 (1), 43-64.

Santiago, Fernando (2014). Innovation for Inclusive Development. Innovation and Development, 4 (1), 1-4.

Sartori, G. (2011). La Política. Lógica y Método de las Ciencias Sociales. México: Fondo de Cultura Económica.

Sørensen, E. y Torfing, J. (2012). Collaborative Innovation in the Public Sector. The Innovation Journal. The Public Sector Innovation Journal, 17 (1), 1-14.

Yin, R. (2004). Case Study Methods. Washington: American Educational Research Association.

Zurbriggen, C. y González, M. (2014). Innovación y Co-Creación: Nuevos Desafíos para las Políticas Públicas. Revista de Gestión Pública, 3 (2), 329-361.

\section{Entrevistas}

Alanís Dávila, E. Presidente del Consejo del Centro de Integración Ciudadana (entrevistado). Rubio, Jesús (entrevistador). 14 de junio del 2016.

Garza Cavazos, E. Director del Centro de Integración Ciudadana. (entrevistado) Rubio, Jesús y Calzada, Marisol (entrevistadores). 31 de mayo del 2016.

Equipo de trabajo del CIC. Cuestionario estructurado aplicado a 8 empleados. Rubio, Jesús y Calzada, Marisol (entrevistadores). Del 31 de mayo al 31 de julio del 2016.

Recibido: 29-03-2016

Aceptación de la versión final: 15-06-2017 